\title{
Study of Viral Load as A Predictive Marker of the Evolution of HIV Type 2 Infection in Burkina Faso
}

\section{Mahamoudou Sanou ${ }^{1,2 *}$, Absatou Ba $/ \mathrm{Ky}^{1,3}$, Assane Neya ${ }^{1}$, Albert T Yonli ${ }^{4}$, Dufrasne François ${ }^{5}$, Jean Ruelle ${ }^{5}$, Benoit Kabamba $^{5}$, Bolni Marius Nagalo ${ }^{6}$, Mamadou Tamboura ${ }^{2}$, Dinanibe Kambiré ${ }^{2}$, Patric Goubau ${ }^{5}$, Rasmata $^{2}$ Traore $^{1,2}$ and Jacques Simporé ${ }^{4}$}

${ }^{1}$ University Joseph KI - ZERBO, Ouagadougou, Burkina Faso

${ }^{2}$ Pediatric University Hospital Charles De Gaulle, Ouagadougou, Burkina Faso

${ }^{3}$ University Hospital Bogodogo Ouagadougou, Burkina Faso

${ }^{4}$ Pietro Annigoni Biomolecular Research Center (CERBA), Department of Biochemistry - Microbiology, University of Ouaga I Prof Joseph Ki ZERBO, Burkina Faso

${ }^{5}$ AIDS Reference Laboratory, AIDS Reference Laboratory Catholic University of Louvain (UCL), Belgium

${ }^{6}$ Department of Hematology/Oncology, Mayo Clinic Scottsdale, AZ, USA

*Corresponding Author: Mahamoudou Sanou, University Joseph KI-ZERBO, Ouagadougou, Burkina Faso.

Received: August 26, 2019; Published: September 20, 2019

DOI: $10.31080 /$ ASMI.2019.02.0378

\section{Abstract}

Background: HIV - 2 infection is characterized by low sexual and vertical transmission and slow clinical and immunological progression. However, it can lead to AIDS. Viral load measurement is a predictive test of the success or failure of antiretroviral therapy. In order to evaluate the efficacy of ARV treatment, we measured plasma viral load; and CD4 T - cell counts in 68 patients, of whom 56 were HIV - 2 infected and 12 were HIV - 1/HIV - 2 co - infected.

Method: We tested EDTA plasma samples stored at $-80^{\circ} \mathrm{C}$, taken from patients followed in sentinel sites in the city of Ouagadougou. The sera were obtained by centrifugation, and the plasmatic viral load quantified by droplet double polymerase chain reaction (dd PCR), at a detection threshold of 10 copies/ml. Sociodemographic, clinical and therapeutic data were collected from patients charts and completed during an interview.

Results: Patients had a mean age of $53.8 \pm 7.8$ years with extremes of [ 38 - 72 years] and were predominantly females (57.4\%), with a sex ratio of 0.80 . There was a predominance of housewives and married couples with respectively $36.8 \%$ and $75.0 \%$ of patients. The majority of patients (77.9\%) were Category A, that is, they were either asymptomatic or in the primary infection phase. $22.0 \%$ of patients were symptomatic, with $13.2 \%$ and $8.8 \%$ respectively belonging to categories B and $\mathrm{C}$. The most observed opportunistic infections were shingles found in $7.3 \%$ of patients, oral candidiasis found in 5.9\%, signs of weight loss (undernutrition) in $5.9 \%$ and genital herpes in $1.5 \%$ of patients. Four patients (4.7\%) had hepatitis B. One case of tuberculosis (1.5\%) was reported. The therapeutic combination of protease inhibitors (Lopinavir / ritonavir) was the most prescribed (94,1\% of patients); $58.8 \%$ of the patients had a CD4 level $<500 / \mathrm{mm} 3$, and $41.2 \%$ had CD4 level $\geq 500$ cells $/ \mathrm{mm} 3$. Plasma viral load was undetectable ( $\leq 10 \mathrm{copies} / \mathrm{ml}$ ) in $70.6 \%$ of patients; $7.3 \%$ had a viral load of 10 to 50 copies / $\mathrm{ml}$, and $19.1 \%$ of patients had a high viral load ( $\geq 101$ copies / ml).

Our study showed that the highest CD4 levels are observed in patients with undetectable viral load (<10copies / mL). This established that the CD4 cell count and the plasma viral load value move in the opposite direction, and are two predictors of the evolution of HIV infection, and the virological and / or immunological response to HAART.

Keywords: HIV-2; Viral Load; CD4 T Cells; Antiretroviral

\section{Introduction}

With 36.7 million people infected worldwide and 95,000 PVHIV in Burkina Faso in 2016, 4\% of them with HIV - 2 infection is a public health priority $[1,2]$.
Belonging to the sub familyorthoretrovirinae, and to the genus Lentivirus. HIV - 2 has a more limited spread and is less pathogenic than HIV - 1 [3]. 
Mainly present in West Africa, HIV - 2 is characterized by its low sexual and vertical transmission and slow clinical and immunological progression [4,5].

The successful scaling up of ART as a global response to the epidemic has made viral load surveillance a new challenge in the management of PV - HIV [6,7].

The measurement of plasma viral load is therefore a key consideration in the follow-up and management of these patients.

It is a predictive test of the success or failure of antiretroviral therapy [8-10].

HIV viral load is the number of RNA copies of the virus per milliliter of blood.

Many investigations have reported HIV - 2 resistance to most of drugs used for its treatment, demonstrating the importance of regular monitoring of the response to treatment in people with HIV infection [8,11-15,].

The WHO recommends that CD4 T lymphocyte count and plasma viral load be available for efficient monitoring.

In our context in Burkina Faso, although there are HIV - 1 viral load assay techniques, HIV-2 infected patients face a biological monitoring problem due to the lack of a viral load quantification technique $[4,10]$.

The aim was to study the plasma viral load of patients infected by HIV type 2 in Burkina Faso as an element of their biological follow - up.

For this reason, we have proposed to measure CVP in HIV - 2 patients, in collaboration with the LRS/UCL (Belgium), in order to assess the relationship between the value of viral load and the efficacy of antiretroviral therapy, and thus the evolution of HIV - 2 infection.

\section{Materials and Methods}

Our study was held in the city of Ouagadougou and at the LRS/ UCL.

The Medical Centre of the General Sangoule Lamizana Military Camp and the associations for the treatment of Pvhiv served as sample collection sites for us.

The laboratories of the CHUP - CDG and CERBM - Pietro Annigoni provided us with the framework for the analysis and storage of our samples.
This was a descriptive prospective study that was conducted from January 2017 to March 2018.

Our study population consisted of women, adult men infected with HIV - 2 or co - infected with HIV - 1 + 2, followed by the associations of PV - HIV in Ouagadougou City and the CMGASL Medical Centre.

Included in our study were any adult HIV2 or HIV1 +2 patients who were members of PEC associations or who were followed at CMGASL on ART.

Socio - demographic characteristics (age, sex, occupation, marital status); clinical (pathologies and associated opportunistic infections); biological (CD4 count, viral load) and therapeutic (ARV, treatment of opportunistic infections) were studied.

The data collected on an individual collection sheet were entered on a microcomputer and analyzed using the EPI - INFO version 7.2.2.2.2, Microsoft Word and Excel 2013 statistical software.

Only HIV - 2 or co - infected patients who consented to participate in the study were selected for sampling.

After checking the serological status, we collected blood from 2 EDTA tubes at the fold of the elbow.

The blood collected was stored in a box and then sent to the laboratory. In the laboratory, after a serological status check, using the Immunocombs II technique, we first deposited 4 drops of blood from each sample on DBS paper.

Then we centrifuged the samples at $3000 \mathrm{rpm}$ for 15 minutes.

After centrifugation, the sera were collected in cryotubes, placed in a cryobox, and stored at $-80^{\circ} \mathrm{C}$.

Finally, the samples and DBS papers were sent to the AIDS Reference Laboratory of the Catholic University of Louvain (LRS/UCL) where the confirmation of the type of HIV and the measurement of the viral load were performed.

Information collected from patients and test results were kept confidential.

\section{Results and Discussions \\ The serology}

At the end of our analysis, we had 85 patients, including 52 HIV - 2 patients and 33 co - infected patients. However, the AIDS reference laboratory found different serological results. Indeed, 13 of 
the patients screened for HIV - $1+2$ co - infection in Burkina Faso were found to be HIV - 1 in Belgium; 4 patients screened for HIV $1+2$ in Burkina Faso were found to be HIV - 2 in Belgium.

In total, our study enrolled 68 patients, 56 of whom were HIV - 2 patients $(82.4 \%)$ and 12 patients living with HIV $-1+2$ co - infection $(17.6 \%)$.

\section{Socio-demographic characteristics}

The average age of the patients was 53.8 ranging from 38 and 72 years old. The most represented age group was [50;60].

We noted a female predominance at $57.4 \%$, or a sex ratio of 0.80 .

The majority of patients (75.0\%) were married.

\section{Clinical and therapeutic characteristics}

Shingles, candidiasis were the most dominant OIs, with $7.3 \%$ and $5.9 \%$ of cases respectively. Most patients (77.9\%) were at clinical stage I.

ART based on Zidovudine + Lamivudine + Lopinavir/Ritonavir was the most prescribed, accounting for $54.4 \%$ of cases.

\section{Biological characteristics}

The majority of patients (48.5\%) had CD4 levels between [200 499 cells/mm3]. And $58.8 \%$ of patients had CD $4<500$ cells $/ \mathrm{mm} 3$ levels.

At the 10 copy $/ \mathrm{ml}$ threshold, $70.6 \%$ of patients had an undetectable viral load.

Most of these patients (with undetectable viral load) had CD4 levels $\geq 500$ cells $/ \mathrm{mm} 3$.

The majority of patients with undetectable viral load nearly $36.8 \%$ of patients, had a treatment duration $\leq 5$ years.

The majority of patients (33.8\%) with CD4 levels $\leq 500$ cells/ $\mathrm{mm} 3 \mathrm{had}$ a treatment duration $\leq 5$ years.

We found differences between the serological diagnoses made in Burkina Faso and Belgium. In 2015, SANOU M. (Burkina) also noted discrepancies between the diagnoses established in Burkina and the LRS/UCL [10].

These discrepancies could probably be explained by the existence of cross - reactions due to the homology between the 2 types of viruses.
These results highlight the importance of having quality reagents for confirmatory serological diagnosis before ARV treatment is initiated.

\section{Socio-demographic characteristics}

The average age reported in our study was 53.8 years. Oumar F. (Mali, 2009), Didier., et al. (2013) and Gottlieb., et al. found mean ages lower than those in our study, at $46.2 ; 45.3$ and 36.9 years respectively [18].

The average age found in patients in our study was between [50 60 years]. This could be explained by the fact that nowadays, with the availability of ARVs and access to care services, Plwha have a longer lifetime, justifying the existence of elderly patients living with HIV.

There was a female predominance. The same observation was made by Didier., et al. who reported a female predominance of $56 \%$; Sanogo M. and Oumar F. also reported a predominance of $58.8 \%$ and $60 \%$ respectively, and a respective sex ratio of 0.68 and $0.66[18,19]$.

The high female prevalence in our study is explained by the high female demographics in Burkina Faso, as well as the vulnerability and high susceptibility of women to contracting HIV [4,10].

Almost all of our patients were married. The same observation was made by Oumar F. and Sanogo M. who found 77.1\% and 63.5\% of married respectively [18].

These results can be explained by the African context in which marriage is often a social requirement $[10,18]$.

\section{Clinical characteristics}

The majority of patients were in clinical stage I, i.e., either asymptomatic or in the primary infection phase.

This high prevalence of asymptomatic patients could be explained on the one hand by the particularly slow clinical course of HIV - 2 and on the other hand by a good response to antiretroviral treatment.

The most common opportunistic infections were shingles and Candidiasis. A similar observation was made by Oumar F. (Mali) which found a predominance of Candidiasis and Shingles with $5.7 \%$ and $8.6 \%$ respectively $[18,19]$.

In addition, N'Dour., et al. (Dakar) and Eholie (Côte d'Ivoire) reported that Candidiasis, Shingles and Tuberculosis were the most common OIs among HIV - 2 patients [20,21]. 
Among the prescribed ARVs, AZT + 3TC $+\mathrm{LPV} / \mathrm{r}$ was the most commonly used. Our results are similar to those of Didier K., et al. who reported that $84.0 \%$ of HIV - 2 patients were on a PI - based diet.

The high use of PIs in our study is explained by the fact that PIs, in this case LPV/r, are recommended for the management of HIV 2 and HIV - $1 / 2$ co - infected patients.

The majority of patients had an undetectable viral load at the threshold of 10 copies $/ \mathrm{ml}$. This indicates good control of infection with antiretroviral therapy.

At the 10 copy/ml threshold, viral load was detectable in only $29.4 \%$ of patients; however, it was detectable in $41.6 \%$ of patients in the study by Didier K., et al.

The detectable viral loads reported in our study could be explained either by the fact that these patients had just started ART, or by poor compliance with treatment, or the existence of resistant strains.

This establishes that the initiation of effective ARV treatment allows HIV - 2 infection to be controlled and significantly reduces plasma viral load.

The analysis of these results showed us that plasma viral load and CD4 T - cell level would be inversely proportional.

In addition, they would depend on whether or not ARV treatment was initiated, the duration, effectiveness of treatment, and the sensitivity of the HIV strain to treatment.

Our results are in accordance with the literature data according to which viral load and TCD4 lymphocyte rate change inversely $[8,13,15,17]$.

Indeed, this hypothesis was also confirmed in studies by Ariyoshi., et al. Berry., et al. (Gambia) reported in 2012 that the viral load of HIV - 2 was even more undetectable as the CD4 cell count increased.

\section{Conclusion}

32 years after its discovery, HIV infection still remains a problem.

In Burkina Faso, considerable progress has been made in the fight against HIV since the first cases were discovered in 1986.

However, the absence of an available test for measuring HIV 2 plasma viral load hinders the monitoring of the effectiveness of antiretroviral therapy in HIV patients2.

\begin{tabular}{|c|c|c|c|c|}
\hline \multirow{2}{*}{ Sex } & \multirow{2}{*}{ Effective } & \multirow{2}{*}{$\%$} & \multicolumn{2}{|c|}{ Type of VIH } \\
\hline & & & VIH-1+2 & VIH-2 \\
\hline Female & 39 & 57,4 & 7 & 32 \\
\hline Male & 29 & 42,6 & 5 & 24 \\
\hline Total & 68 & 100,0 & 12 & 56 \\
\hline \multicolumn{5}{|l|}{ Sex-ratio: 0,8} \\
\hline Married & 51 & 75.0 & 9 & 42 \\
\hline Single & 6 & 8,8 & 0 & 6 \\
\hline Common union & 5 & 7,3 & 1 & 4 \\
\hline Divorced) & 5 & 7,3 & 1 & 4 \\
\hline Widower & 1 & 1,5 & 1 & 0 \\
\hline Total & 19 & 100,0 & 12 & 56 \\
\hline \multicolumn{5}{|l|}{ ARV } \\
\hline $\begin{array}{l}\text { AZT / 3TC / } \\
\mathrm{LPV} / \mathrm{r}\end{array}$ & 37 & 54,4 & 4 & 33 \\
\hline $\begin{array}{l}\mathrm{TDF} / 3 \mathrm{TC} / \\
\mathrm{LPV} / \mathrm{r}\end{array}$ & 18 & 26,5 & 3 & 15 \\
\hline $\begin{array}{l}\mathrm{TDF} / \text { FTC / } \\
\mathrm{LPV} / \mathrm{r}\end{array}$ & 8 & 11,7 & 3 & 5 \\
\hline $\mathrm{ABC} / 3 \mathrm{TC} / \mathrm{LPV} / \mathrm{r}$ & 1 & 1,5 & 0 & 1 \\
\hline $\begin{array}{l}\text { AZT / 3TC / DR- } \\
\mathrm{V} / \mathrm{r}\end{array}$ & 1 & 1,5 & 0 & 1 \\
\hline AZT / 3TC / EFV & 1 & 1,5 & 1 & 0 \\
\hline TDF/3TC/NVP & 1 & 1,5 & 1 & 0 \\
\hline Autres & 1 & 1,5 & 0 & 1 \\
\hline TOTAL & 6 & 100 & 12 & 56 \\
\hline \multicolumn{5}{|l|}{ Viral load } \\
\hline$<10$ & 48 & 70,6 & 12 & 36 \\
\hline$[10-50]$ & 5 & 7,3 & 0 & 5 \\
\hline$[50-100]$ & 2 & 2,9 & 0 & 2 \\
\hline [100 - 1000] & 11 & 16,2 & 0 & 11 \\
\hline$[1000-45000]$ & 2 & 2,9 & 0 & 2 \\
\hline TOTAL & 68 & 100,0 & 12 & 56 \\
\hline $\mathrm{P}=0,19$ & & & & \\
\hline
\end{tabular}

Table 1: Socio-demographic, biological and therapeutic data of patients.

\begin{tabular}{|l|c|c|c|c|}
\hline \multicolumn{1}{|c|}{ Viral load } & \multicolumn{3}{|c|}{ CD4 count } & Total \\
\hline & {$[\mathbf{0}-\mathbf{2 0 0}]$} & {$[\mathbf{2 0 0 - 4 9 9 ]}$} & $\mathbf{2 5 0 0}$ & \\
\hline$<10$ & 2 & 13 & 21 & 36 \\
\hline$[10-50]$ & 1 & 2 & 2 & 5 \\
\hline$[50-100]$ & 0 & 2 & 0 & 2 \\
\hline$[100-1000]$ & 3 & 8 & 0 & 11 \\
\hline$[1000-45000]$ & 1 & 1 & 0 & 2 \\
\hline TOTAL & 7 & 26 & 23 & 56 \\
\hline
\end{tabular}

Table 2: Correlation between viral load and CD4 T Lymphocyte count in HIV-2 patients. $P=0,05$ 
Our study allowed us to perform confirmatory serological diagnosis tests in 68 HIV - 2 patients, quantify their plasma viral load for the first time since initiation of their treatment, and evaluate the efficacy of antiretroviral therapies in these patients.

It appears that the serological tests carried out in the monitoring centers are not always reliable for the initiation of ARV treatment. This requires quality control.

In addition, combining CD4 $\mathrm{T}$ cell count and quantification of the highly sensitive HIV - 2 viral load with a threshold of 10 copies/ mL could improve the initiation and monitoring and evaluation of patients' ART.

Therefore, the implementation of the HIV2 viral load measurement technique in Burkina Faso is necessary for regular follow - up of HIV - 2 patients as well as HIV 1.

\section{Bibliography}

1. Unaids_Burkina Faso. Report on The Hiv/Aids Epidemic (2016).

2. National Multisectoral Plan to Fight Hiv, Aids and Ist Annee (2017).

3. Aubry P. Infection par le VIH/Sida et tropiques; Médecine Tropicale Diplôme de médecine tropicale des pays de l'Océan Indien (2017).

4. Ruelle J., et al. "Genetic Polymorphisms and Resistance Mutations of HIV Type 2 in Antiretroviral-Naive Patients in Burkina Faso". AIDS Res Hum Retroviruses 23.8 (2007): 955-964.

5. Kanki PJ., et al. "Slower heterosexual spread of HIV-2 than HIV-1". Lancet Lond Engl 343.8903 (1994):943-946.

6. Ntemgwa ML., et al. "Antiretroviral Drug Resistance in Human Immunodeficiency Virus Type 2". Antimicrob Agents Chemother 53.9 (2009): 3611-3619.

7. Brower ET., et al. "Inhibition of HIV-2 Protease by HIV-1 Protease Inhibitors in Clinical Use". Chemical Biology and Drug Design 71.4 (2008): 298-305.

8. Consolidated Guidelines on the Use of Antiretroviral Drugs for Treating and Preventing HIV Infection: Recommendations for a Public Health Approach (2016).

9. Gottlieb GS., et al. "Emergence of Multiclass Drug-Resistance in HIV-2 in Antiretroviral-Treated Individuals in Senegal: Implications for HIV-2 Treatment in Resouce-Limited West Africa". Clinical Infectious Diseases 48.4 (2009): 476-483.

10. Mahamadou S. Problematique Du Vih Au Burkina Faso: Securite Transfussionelle, Polymorphisme Genique Et Resistance Au Traitement Du Vih-2. [Laboratoire de Biologie Moléculaire]: Universite De Ouagadougou (2015).
11. Pascal N., et al. Normes Et Protocoles De Prise En Charge Medicale Des Personnes Vivant Avec Le Vih Au Burkina Faso. Pssls/ Ist. (2017).

12. IeDEA. Characteristics of HIV-2 and HIV-1_HIV-2 Dually Seropositive Adults in West Africa Presenting for Care and Antiretroviral Therapy_The IeDEA-West Africa HIV-2 Cohort Study. html.

13. Gottlieb GS., et al. "Equal Plasma Viral Loads Predict a Similar Rate of CD4 + T Cell Decline in Human Immunodeficiency Virus (HIV) Type 1- and HIV-2-Infected Individuals from Senegal, West Africa". Journal of Infectious Diseases 185.7 (2002):905-914.

14. Ekouévi DK., et al. "Plasma HIV-2 RNA According to CD4 Count Strata among HIV-2-Infected Adults in the IeDEA West Africa Collabor".

15. Berry N., et al. "HIV type 2 proviral load measured by quantitative polymerase chain reaction correlates with CD4+ lymphopenia in HIV type 2-infected individuals". AIDS Res Hum Retrovir 10.8 (1994): 1031-1037.

16. Descamps D. "Infections VIH: Outils Virologiques Diagnostic de l'infection Quantification virale Résistance”. GH Bichat-Claude Bernard Inserm Umr 1137 Université Paris-Diderot Sorbonne Paris cité (2015).

17. Egger M., et al. "Prognosis of HIV-1-infected patients starting highly active antiretroviral therapy: a collaborative analysis of prospective studies". Lancet 360.9327 (2002):119-129.

18. Oumar F. L'infection Par Les Vih1-Vih2 Associes Dans Les Chu Gabriel Toure Et Du Point «G» De Bamako (2009).

19. Sanogo M. Enquete Sero-Epidemiologique Sur L'infections Par Les Vih Au Cesac De 2001 A 2003. [Faculté de Médecine, de Pharmacie et d'Odonto-Stomatologie]: Université de Bamako (2004).

20. Fortes Déguénonvo L., et al. "Profil actuel des patients infectés par le VIH hospitalisés à Dakar (Sénégal)". Bulletin de la Société de Pathologie Exotique 104.5 (2011): 366-370.

21. Eholie SP., et al. "Epidemiological and clinical features of HIV2 infection in Abidjan, Ivory Coast". Medicina Tropical 58.2 (1998): 204-205.

\section{Volume 2 Issue 10 October 2019} (C) All rights are reserved by Mahamoudou Sanou., et al. 